\title{
ARTÍCULOS
}

\section{TRUMP VS LOS MEDIOS. TRATAMIENTO DE LA PRENSA DESDE LA CUENTA DE TWITTER DEL PRESIDENTE DE EUA}

\author{
Trump vs. media. Treatment of the press from the US \\ president's Twitter account
}

\section{Elena Yeste y Pere Franch}

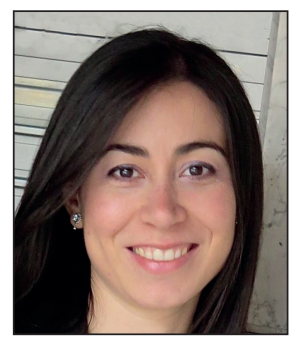

Elena Yeste es periodista y doctora por la Universitat Ramon Llull (URL). Profesora de Comunicación y coordinadora del Master Universitario en Comunicación Política y Social y del Master Universitario en Periodismo Avanzado-Reporterismo de la Facultad de Comunicación y Relaciones Internacionales Blanquerna. Redactora jefa de Trípodos. Redactora del magazín La mira. Fue redactora de Avui y del programa Afers Exteriors de TV3. Autora de L'era de la hipermemòria, Premio Josep Vallverdú de Ensayo (Pagès, 2010), y de Un conflicte de llengües, Premio Ramon Trias Fargas de Ensayo Político (Angle, 2015).

https://orcid.org/0000-0002-3383-316X

elenayp@blanquerna.url.edu

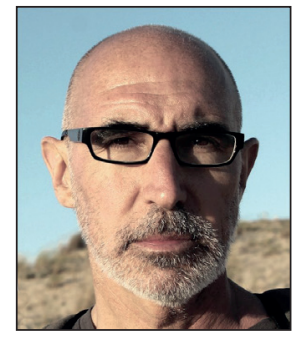

Pere Franch, doctor por la Universitat Ramon Llull (URL), es profesor de Periodismo y de Relaciones Internacionales en la Facultad de Comunicación y Relaciones Internacionales Blanquerna de la URL, y coordinador del Departamento de Periodismo de la misma Facultad. Director del Master en Periodismo Internacional, ha trabajado en radio (COM Ràdio) y prensa escrita (El observador y Avui). Autor de los libros La guerra des de la Casa Blanca, Premio Josep Vallverdú de Ensayo (Pagès, 2013) y La falacia de la libertad (Brumaria, 2018). https://orcid.org/0000-0002-4180-1168

perefp@blanquerna.url.edu

Universidad Ramon Llull, Facultad de Comunicación y Relaciones Internacionales Blanquerna Plaza Joan Coromines, s/n. 08001 Barcelona, España

\section{Resumen}

Este artículo examina el tratamiento del presidente Donald Trump en Twitter hacia los medios de comunicación norteamericanos durante sus primeros seis meses de mandato, del 20 de enero al 20 de julio de 2017. A partir de un análisis de contenido de los tweets publicados en su cuenta @realDonaldTrump, se estudian las menciones a los medios y el tono de dichas menciones, y la presencia y el uso de las palabras clave relacionadas con los medios. Asimismo, se analiza el tratamiento de Trump hacia la prensa en relación con el control político y la función del periodismo como perro guardián (watchdog) de la democracia. Como conclusiones principales se constata que Trump, de manera muy simple y directa, elogia a los medios afines y critica a los que según él le son hostiles, a los que muy a menudo califica de fake news. Con ello, Trump sortea el papel mediador de los medios y niega la misión de control del poder político que se les atribuye en los sistemas democráticos.

\section{Palabras clave}

Twitter; Medios; Redes sociales; Periodismo; Mediación periodística; Watchdog; Control político; Populismo; Donald Trump.

\section{Abstract}

This article examines President Donald Trump's treatment on Twitter towards the US media during his first six months in office, from January 20 to July 20, 2017. Based on a content analysis of the tweets published in the account @realDonaldTrump, we study the mentions to the media and their tone, and the presence and use of keywords related to the media. In addition, we analyze Trump's treatment of the press in relation to political control and the role of journalism as watchdog of democracy. As main conclusions it is noted that Trump, in a very simplistic way, praises those media that treat him positively and criticizes those that -in his view- portrait him negatively, which are often dubbed as fake news. Through this process, Trump avoids the mediator role of the media and rejects the watchdog role that democratic systems have attributed to them.

\section{Keywords}

Twitter; Media; Social media; Journalism; Mediation; Watchdog; Political control; Microblogging; Populism; Donald Trump. 
Yeste, Elena; Franch, Pere (2018). "Trump vs los medios. Tratamiento de la prensa desde la cuenta de Twitter del presidente de EUA". El profesional de la información, v. 27, n. 5, pp. 975-983.

https://doi.org/10.3145/epi.2018.sep.02

\section{Introducción}

El mismo día de su toma de posesión como 44ํ presidente de los Estados Unidos, el 20 de enero de 2017, Donald Trump se enzarzó en una nueva polémica con los periodistas: su equipo de prensa informó de que su ceremonia de juramento había sido la más concurrida de la historia de Estados Unidos. Ante la evidencia de las fotografías que demostraban mucha menor afluencia que en la toma de posesión de Barack Obama en 2009, su jefe de prensa, entonces Sean Spicer, declaró que los "deshonestos" medios ofrecían imágenes gráficas que distorsionaban intencionadamente la realidad. Dos días después, la consejera presidencial Kellyanne Conway declaró que la Casa Blanca presentaba "hechos alternativos" a las cifras que daba la prensa; y Donald Trump, en relación con las informaciones sobre su enfrentamiento con la $C I A$, declaró que los periodistas están "entre los seres humanos más deshonestos del mundo".

El enfrentamiento de Trump con los medios de comunicación que no le son afines se ha evidenciado en diversos sitios, pero donde más prolíficamente ha desatado sus iras contra ellos ha sido en la red social Twitter. A través de su cuenta oficial, @RealDonaldTrump, el presidente tiene acceso directo a sus (en mayo de 2018) más de 51 millones de seguidores. Desde marzo de 2009 ha publicado 37.500 tweets, lo que supone una media de más de 11 tweets diarios.

El presente estudio analiza los tweets publicados por Trump en su cuenta durante su primer medio año de mandato, del 20 de enero al 20 de julio de 2017, con el objetivo de observar qué trato dispensa a través de esta red a los medios de comunicación y a los periodistas. Se ha analizado el contenido de los 1.006 tweets publicados en ese período y se ha observado en cuántos de ellos se hacía una referencia, directa o no, a los medios de comunicación, qué decía, en qué términos, en qué contexto y con qué tono.

En el proceso de análisis se han tomado en consideración las relaciones entre el poder y los medios y el papel que tienen éstos -y en especial las redes sociales- en la conformación de la percepción de la realidad por parte de los ciudadanos.

\section{Marco teórico y estado del arte}

Han pasado casi tres lustros desde que en 2004 Tim O’Reilly acuñara el término web 2.0 para referirse a las aplicaciones digitales que iban a proporcionar a los usuarios de internet la posibilidad de interactuar, compartir información y colaborar entre ellos. Una realidad que la aparición de Twitter en 2006, junto al resto de redes sociales, generalizó y extendió por todo el mundo. La proliferación y el creciente protagonismo de las redes dio pie a la conocida expresión de Castells "autocomunicación de masas" (Castells, 2009), ya que estas redes permiten a los ciudadanos autoseleccionar, autoproducir y autodistribuir sus propios contenidos.
Las redes sociales constatan que la comunicación de masas ha dejado de ser unidireccional y vertical: la teoría clásica de la aguja hipodérmica quedó superada y estamos de lleno en la era de la comunicación digital, que es interactiva, multimediática, hipertextual y multidireccional. Sin embargo, no por ello dejan de ser aplicables hoy en día algunos de los paradigmas teóricos clásicos de la comunicación social. Para el presente estudio entendemos que también las redes sociales tienen capacidad de construcción de la realidad social que la escuela constructivista atribuye a los medios de comunicación convencionales (Berger; Luckmann, 1967). El intenso uso que Donald Trump hace de Twitter pone de manifiesto, entendemos, su voluntad de influir en la construcción de la percepción de la realidad de sus seguidores.

Los dirigentes políticos han tomado buena cuenta de la capacidad de influencia que, gracias a su elevado número de usuarios, tienen las redes sociales. Twitter es la cuarta red social más utilizada del mundo, tras Facebook, YouTube e Instagram, según el último informe del Reuters Institute (Newman et al., 2018). Esta red pasó de 30 millones a 330 millones de usuarios entre enero de 2010 y octubre de 2017, según datos de Twitter. La misma compañía informó en octubre de 2017 que en Estados Unidos tenía 69 millones de usuarios.

Desde el éxito de Twitter en la campaña de Barack Obama de 2008 (Gainous; Wagner, 2014), el empleo de esta plataforma ha proliferado entre los dirigentes políticos de todo el mundo. Y el uso que han hecho de ella se ha intensificado durante los períodos electorales, tema en el que se ha centrado la mayor parte de las investigaciones académicas sobre el uso de esta red social por parte de los políticos. A los primeros estudios que se realizaron (Amman, 2010; Golbeck; Grimes; Rogers, 2010; Aharony, 2012; Adams; McCorkindale, 2013), cabe añadir el amplio trabajo de Jungherr (2016), que se hace eco de las conclusiones de 127 estudios realizados en 26 países. Dicha investigación agrupa los temas tratados en tres áreas:

- uso de la red por parte de los partidos y los candidatos;

- uso por parte de los públicos;

- su relación con las informaciones de los medios.

Respecto a la primera categoría, que es la que interesa para este estudio, las investigaciones llegan a la conclusión de que, en general, los partidos y los candidatos tienden a utilizar Twitter para divulgar información sobre sus actos de campaña y para publicar enlaces a sus propias webs (Graham; Jackson; Broersma, 2016; Small, 2011). Se pone de manifiesto también que es muy poco frecuente que los políticos publiquen mensajes con contenido personal, lo que lleva a la conclusión de que hacen de esta red social un uso divulgativo, difusor de información ("broadcasting style", dice Jungherr), ya que es prácticamente inexistente la interacción con el resto de usuarios. Con esto se refuer- 
za la comunicación unidireccional del líder hacia el público, pero no viceversa (Adams; McCorkindale, 2013; Aharony, 2012; Enli; Skogerbø, 2013; Jackson; Lilleker, 2011). Por tanto,

"hay poca evidencia de que Twitter sea un instrumento que facilite el diálogo entre los candidatos y los ciudadanos corrientes" (Jungherr, 2016, p. 76).

En España, algunos de los estudios más recientes destacan el poder emocional y afectivo que pueden tener los mensajes de Twitter (Coromina, 2017) o la efectividad de compartir aspectos personales de la vida de los candidatos para conseguir mayor difusión, como constata el uso que el colectivo La Cueva hizo de Twitter para promocionar al candidato de Izquierda Unida, Alberto Garzón, en las elecciones de 2015 y 2016 (Hernández-Santaolalla; Rubio-Hernández, 2017).

En cuanto al uso de Twitter por parte de Trump, cabe reseñar el reciente trabajo de Stolee y Caton (2018) sobre el lenguaje con el que se dirigió el presidente a su "base" para conseguir la victoria, una acción de "hablar" a sus votantes que se realizó sobre todo a través de Twitter, con su estilo y su peculiar insistencia, lo que supone un cambio de paradigma en la comunicación del presidente con sus votantes.

Es destacable también el estudio de Enli (2017), que reafirma que en la campaña de 2016 los dos candidatos utilizaron las redes sociales no sólo para sortear a los medios convencionales y dirigirse directamente a sus eventuales electores, sino para debatir el papel y criticar la acción de los mainstream media. En su trabajo, Enli compara las campañas demócrata y republicana y constata que mientras la de Clinton conservó cierta profesionalización, la de Trump se caracterizó por un estilo más amateur pero a la vez más "auténtico", lo que apuntaría hacia una tendencia contraria a la profesionalidad y a favor de la espontaneidad en la comunicación política.

En la misma línea, Ott (2017) plantea su estudio de caso sobre la cuenta de Twitter de Trump partiendo de la base y corroborando que esta red social privilegia el discurso "simple, impulsivo e irrespetuoso".

En este contexto, cabe tener en cuenta también los estudios previos sobre el control político del periodismo, que abordan

"la fiscalización periodística sobre la política, vinculada a la teoría del watchdog, y las presiones sobre los medios ejercidas por el sistema político" (Casero-Ripollés; Izquierdo-Castillo; Doménech-Fabregat, 2014, p. 25).

\section{Objetivos e hipótesis}

El objetivo de este artículo es analizar el tratamiento que el presidente de Estados Unidos, Donald Trump, da a los medios de comunicación norteamericanos en los primeros seis meses de su mandato. Particularmente, el artículo se propone estudiar el contenido de los mensajes publicados por el presidente Trump en la red social Twitter, tanto los retweets como los tweets generados, con el fin de explorar su relación con la prensa y con los principales grupos mediáticos en su primer medio año de mandato. En este sentido:

- se examina el tono de los tweets del mandatario norteamericano;

- se contextualizan dichos contenidos en el marco de las informaciones y de los acontecimientos políticos en que se han producido;

- se analiza la presencia, el uso y el carácter de las palabras clave relacionadas con los medios periodísticos.

La principal hipótesis de trabajo es que la tendencia de su relación con los medios de comunicación estadounidenses es marcadamente negativa, y su estrategia comunicativa en relación con los medios muy focalizada hacia los medios críticos con su gestión política y especialmente desacreditadora con su tarea informativa, a quienes acusa y señala directamente como fake news ofake news media.

Este estudio pretende identificar el número de menciones realizadas a medios de comunicación y grupos mediáticos y valorar su tono. A la vez, se presume que las alusiones a los medios considerados como afines presentan un tono positivo. De esta manera, el uso y la estrategia comunicativa de Trump en Twitter estaría orientada a desmentir las informaciones políticas de medios considerados no afines y afianzar su posición política, con el uso de un tono peyorativo o menospreciativo que mina, cuestiona y contraviene la función del periodismo como perro guardián o watchdog.

\section{Metodología}

El presente estudio se fundamenta en un análisis de con- 
tenido a partir de técnicas cuantitativas. Partiendo de la grounded theory o teoría fundamentada en datos (Miles; Huberman, 1994; Corbin; Strauss, 1990), se ha realizado un análisis derivado de la observación de la realidad material, específicamente el contenido de los tweets del presidente Trump en sus seis primeros meses de mandato, con el fin de recopilar los datos referidos al tratamiento ofrecido por su parte a los medios, y poder así llegar a conclusiones.

En primer lugar, del conjunto de 1.006 tweets publicados por Trump en su primer medio año presidencial se han seleccionado aquellos que hacen referencia a medios de comunicación, y se han analizado para crear las correspondientes categorías para su posterior estudio. Los medios identificados han sido:

- Fox: con categorías específicamente creadas para Fox News, Fox and Friends y Fox Nation;

- The Drudge Report;

- The New York Times;

- CNN;

- $A B C$

- NBC;

- el programa Morning Joe de la Msnbc;

- The Washington Post;

- CBS;

- una categoría para otros medios, entre los que se incluyen: la Christian Broadcasting Network, The Hill, The Daily Caller, ComCast, Wall Street Journal, The Washington Times, The Gazette;

- una categoría para las referencias a medios en general.

Como parte del análisis de contenido realizado, se ha observado el tono de los tweets a partir de tres categorías: positivo, negativo y neutro, partiendo de un estudio de referencia en este campo (Kahn; Kenney, 2002), con el objetivo de analizar la actitud de Trump en relación con los medios de comunicación aludidos.

Las referencias del presidente a los medios del grupo Fox son siempre positivas o neutras

Finalmente, se ha llevado a cabo un estudio de las palabras clave utilizadas en la muestra. A partir de la observación del contenido de los tweets, se ha identificado una serie de términos con los que el presidente Trump se ha referido en sus tweets a la información periodística y, en particular, a los medios de comunicación estadounidenses. El resultado de este análisis ha concluido en la definición del siguiente conjunto de palabras clave:

- fake news (con todas las combinaciones posibles: fake, fake news media, fake media, fake news writers, mainstream (fake) media, fake MSM, fake \& fraudulent news media);

- failing;

- fraud news;

- fabricated lies o lie;

- dishonest; y

- real news.
Algunas palabras clave aparecen referidas a más de un medio de comunicación, como es el caso del siguiente tweet del 6 de febrero en el que Trump defiende su política antiinmigración, donde el término fake news alude a varios me$\operatorname{dios}(C N N, A B C$ y $N B C)$ :

"Any negative polls are fake news, just like the $C N N, A B C$, $N B C$ polls in the election. Sorry, people want border security and extreme vetting".

En este caso, la palabra clave se contabiliza una vez, pero se contabilizan tres menciones a medios.

\section{Resultados}

De los 1.006 tweets que Donald Trump publicó en el período estudiado, más de una quinta parte, 212 (21\%), contienen una o varias referencias a algún medio de comunicación en particular o a los medios en general. Cabe destacar que del total de tweets recuperados, el $76,42 \%$ fueron generados por el propio presidente frente a un $23,58 \%$ de contenidos retweeteados.

Hemos distinguido entre los que le son afines y los que -a su entender- le son hostiles. Entre los primeros, el más citado es el grupo Fox: de entre las 74 menciones, cabe distinguir las de la cadena Fox News (22) y sobre todo las de su magazine matinal Fox and Friends (43 menciones). En el caso de este programa, en la mayoría de los casos el tono empleado es neutro porque Trump se limita a retweetear mensajes: eso ocurre en 28 de los 43 casos. Cuando no hace un retweet, simplemente informa de algo que se ha dicho, ha ocurrido o va a ocurrir en el programa, como por ejemplo cuando el 12 de junio informaba de que

"My daughter, Ivanka, will be on @foxandfriends tomorrow morning. Enjoy!".

Aunque hay menos retweets de Fox News, en la mayoría de los casos el tono también es neutro. En los casos en que es positivo, Trump elogia directamente afirmaciones emitidas, la programación de esta cadena, la cobertura de un tema o sus índices de audiencia. Las cinco referencias a Fox son en realidad tweets en los que menciona programas suyos e informa de que será entrevistado por alguno de sus periodistas estrella: Sean Hannity, Lou Dobbs o Maria Bartiromo. Y en el caso de Fox Nation, las cuatro referencias son retweets de la cuenta de Twitter de esa web. Si bien el tono respecto a este medio web es neutral, el contenido de los tweets es siempre positivo para Trump, como por ejemplo cuando el 12 de abril retweetea:

“Grateful Syrians React To @RealDonaldTrump Strike:

'I'll Name My Son Donald'”.

Este retweet refleja que Trump destaca que se le elogie más a él que a su acción, como queda ejemplificado en el hecho de que un agradecido ciudadano sirio quiera llamar Donald a su hijo venidero después del ataque norteamericano con misiles contra la base aérea de Al Shayrat, el 6 de abril de 2017.

También es afín a Trump The Drudge Report, una web conservadora que agrega contenidos de otros medios, cuyo contenido Trump retweetea en seis ocasiones. Es destacable que otro medio supuestamente afín como Breitbart news, 
dirigido por Stephen K. Bannon, que fue su jefe de campaña y hasta agosto de 2017 jefe de estrategia de la Casa Blanca, no aparece ni una sola vez en los más de mil tweets publicados en el primer medio año de presidencia de Trump.

En cuanto a los medios que no le son afines, Trump no publica ni un sólo tweet en tono positivo: sólo en seis casos es neutro y en el resto de las referencias es siempre negativo.

Atención especial merecen el periódico The New York Times y la cadena $C N N$, principales focos de las iras del presidente.

Las 23 referencias al periódico de Nueva York son todas negativas excepto dos: en una sólo publica el enlace a una información del diario del 17 de marzo que informa de que ha cambiado el grado de confianza de la pequeña empresa y el otro es un retweet del programa Fox and Friends en el que se informa de que el editor pide perdón por un tweet publicado. En cuanto al resto de referencias, en todas ellas menos en cuatro Trump siempre acompaña el nombre del diario con la expresión "failing" -fracasado, fallido.

\section{La mayor parte de menciones a The New York Times van acompañadas de la ex- presión failing - fracasado}

Es destacable que poco después de tomar posesión, Trump dedica siete tweets a repetir que el periódico pidió perdón a sus lectores por la errónea cobertura de su victoria, cuando en realidad el editor publicó una carta a sus suscriptores tras las elecciones de 2016 en la que se preguntaba si

"la total falta de convencionalidad de Trump nos llevó a nosotros y a otros medios a subestimar su apoyo entre los votantes estadounidenses" (Sulzberger; Baquet, 2016).

En otro tweet del 30 de marzo, Trump acusa al periódico de arruinar a todo el sector de la comunicación e incluso llega a plantear el cambio de las leyes que regulan el libelo ante lo que considera una errónea información sobre él:

"The failing @nytimes has disgraced the media world. Gotten me wrong for two solid years. Change libel laws?".

La CNN no sale mejor parada. Las 16 referencias directas a la cadena son todas negativas; en la mayoría se refiere a ella (en algunos casos junto a los otros medios) con el término fake news y la acusa de mentir o de informar de manera tergiversada. Su agresividad con esta cadena llega al paroxismo en dos tweets del sábado 1 de julio: en uno dice:

"I'm thinking about changing the name \#FakeNews CNN to \#FraudNewsCNN!".

A las pocas horas publica otro con el texto "\#FraudNews CNN \#FNN" y un enlace a un montaje de vídeo que se hizo viral en el que aparece él durante un combate de lucha (wrestling) agrediendo a un personaje cuya cabeza se ha caracterizado con el logo de la CNN.

El resto de medios que Trump considera hostiles
$(A B C, N B C$, The Washington Post y $C B S)$ también son tratados casi siempre de manera muy negativa (sólo en 4 casos el tono es neutro) y a menudo asociados a lo que él llama fake news media.

Gracias a Twitter, Trump sortea el papel mediador de los media e impide su papel de watchdog del gobierno

Aparte de estas referencias directas a determinados medios, en 68 ocasiones se refiere a los medios en general, siempre de manera muy despectiva, con los términos fake news media, mainstream media, media, o the press, a los que acusa de "mentir", "ser deshonestos", "distorsionar" la realidad, informar de manera parcial o silenciar sus supuestos éxitos. La apreciación de Trump es muy simple: cuando los medios cuestionan sus decisiones y sus políticas, le traicionan a él y al país, con lo que se convierten también en enemigos de los estadounidenses. Por tanto, en su universo mental, quien critica la acción presidencial es contrario a los intereses de los norteamericanos, pero quien está a favor, hace un buen servicio a la patria. En una actitud característica de los políticos populistas, Trump se identifica a sí mismo con el pueblo y quien le contradice es enemigo del pueblo norteamericano. En un tweet del 17 de febrero decía:

“The FAKE NEWS media (failing @nytimes, @NBCNews, @ABC, @CBS, @CNN) is not my enemy, it is the enemy of the American People!".

Frente a los fake news media, Trump reivindica el poder de sus redes sociales como canal de comunicación directo con el público. En un tweet del 16 de junio expresaba:

Tabla 1. Menciones a medios de comunicación y tono en el que son tratados por Trump en su cuenta de Twitter.

\begin{tabular}{|l|c|c|c|c|}
\hline \multirow{2}{*}{ Medios } & \multirow{2}{*}{ Menciones } & \multicolumn{3}{c|}{ Tono } \\
\cline { 3 - 5 } & & Positivo & Neutro & Negativo \\
\hline Fox and Friends & 43 & 4 & 39 & 0 \\
\hline Fox News & 22 & 7 & 15 & 0 \\
\hline Fox & 5 & 2 & 3 & 0 \\
\hline Fox Nation & 4 & 0 & 4 & 0 \\
\hline Total Fox & 74 & 13 & 61 & 0 \\
\hline & & & & \\
\hline The Drudge Report & 6 & 0 & 6 & 0 \\
\hline The New York Times & 23 & 0 & 2 & 21 \\
\hline CNN & 16 & 0 & 0 & 16 \\
\hline ABC & 11 & 0 & 3 & 8 \\
\hline NBC & 11 & 0 & 0 & 11 \\
\hline Morning Joe (Msnbc) & 3 & 0 & 0 & 3 \\
\hline The Washington Post & 8 & 0 & 0 & 8 \\
\hline CBS & 3 & 0 & 1 & 2 \\
\hline Otros & 10 & 1 & 7 & 2 \\
\hline Medios en general & 68 & 0 & 0 & 68 \\
\hline & & & & 139 \\
\hline Total & 233 & 14 & 80 & \\
\hline
\end{tabular}


"The Fake News Media hates when I use what has turned out to be my very powerful Social Media - over 100 million people! I can go around them".

En un tweet previo del 6 de junio, Trump ya aludía al hecho de que los medios sociales permiten una comunicación sin filtros ni mediación periodística:

"The FAKE MSM is working so hard trying to get me not to use Social Media. They hate that I can get the honest and unfiltered message out".

Se han contabilizado 115 menciones a palabras clave en el conjunto de los 212 tweets referidos a medios de comunicación. Ello supone que el $54,25 \%$ de los tweets analizados contiene al menos una palabra clave. Del total de tweets, 81, más de un tercio, utilizan la palabra fake, 17 failing, siete dishonest, cinco fabricated lies/lie, dos real news, dos MSM (del inglés mainstream media), y uno garbage journalism.

El presidente acusa a los medios que no le son afines de "mentir", "distorsionar" y ser "deshonestos"

La segunda palabra clave más utilizada es failing ( 28 veces), vinculada a The New York Times en 17 ocasiones, la CNN (3), The Washington Post (2), los canales $A B C$ (2) y $N B C$ (2), el programa Morning Joe de la Msnbc (1) y la CBS (1).

Los términos más utilizados a continuación son dishonest (10), fabricated lies/lie (5), fraud news (2), real news (2), MSM (2) y garbage journalism (1). El adjetivo dishonest se atribuye en cinco ocasiones a los medios en general, como en este tweet del 2 de julio:

"The dishonest media will NEVER keep us from accomplishing our objectives on behalf of our GREAT AMERICAN PEOPLE!..."
Tabla 2. Palabras clave analizadas en los tweets del presidente Trump

\begin{tabular}{|l|c|}
\hline \multicolumn{2}{|c|}{ Palabras clave } \\
\hline Fake (todas las posibilidades) & 81 \\
\hline Failing & 17 \\
\hline Dishonest & 7 \\
\hline Fabricated lies /lie & 5 \\
\hline Real news & 2 \\
\hline MSM & 2 \\
\hline Garbage journalism & 1 \\
\hline Total & 115 \\
\hline
\end{tabular}

dos veces a The New York Times y una a The Washington Post, como en un tweet del 28 de enero en el que arremete contra ambos medios:

“(...) They got me wrong right from the beginning and still have not changed course, and never will. DISHONEST"

y una vez a la $A B C$ y la $N B C$, respectivamente.

La cuenta de Twitter de Trump acusa a los medios en general de fabricar mentiras o mentir en cinco ocasiones, y en una ocasión a The York Times y la CNN. Trump también se refiere a la CNN como garbage journalism en otro tweet del 1 de julio.

\section{Conclusiones}

El posicionamiento de Trump ante los medios es simple, Ilano y superficial:

- los que le son afines -la mayoría del conglomerado Foxreciben siempre, en términos absolutos, un trato neutro o positivo en sus tweets;

- los que a su entender le son hostiles -el resto, a los que se refiere casi siempre como fake news media, mainstream

Tabla 3. Menciones a medios de comunicación y frecuencia de aparición de palabras clave en los tweets de Trump

\begin{tabular}{|c|c|c|c|c|c|c|c|c|c|}
\hline \multirow[b]{2}{*}{ Menciones a medios } & \multicolumn{9}{|c|}{ Palabras clave } \\
\hline & Fake news & Failing & $\begin{array}{l}\text { Fraud } \\
\text { news }\end{array}$ & $\begin{array}{c}\text { Fabricated } \\
\text { lies/lie }\end{array}$ & Dishonest & Real news & $\begin{array}{c}\text { Garbage } \\
\text { journalism }\end{array}$ & MSM & $\begin{array}{c}\text { Total por } \\
\text { medios }\end{array}$ \\
\hline Fox News & 3 & 0 & 0 & 0 & 0 & 0 & 0 & 0 & 3 \\
\hline Fox & 0 & 0 & 0 & 0 & 0 & 0 & 0 & 0 & 0 \\
\hline Fox and Friends & 0 & 0 & 0 & 0 & 0 & 0 & 0 & 0 & 0 \\
\hline Fox Nation & 0 & 0 & 0 & 0 & 0 & 0 & 0 & 0 & 0 \\
\hline The Drudge report & 0 & 0 & 0 & 0 & 0 & 0 & 0 & 0 & 0 \\
\hline The New York Times & 8 & 17 & 0 & 1 & 2 & 0 & 0 & 0 & 28 \\
\hline CNN & 12 & 3 & 2 & 1 & 0 & 0 & 1 & 0 & 19 \\
\hline$A B C$ & 6 & 2 & 0 & 0 & 1 & 0 & 0 & 0 & 9 \\
\hline$N B C$ & 8 & 2 & 0 & 0 & 1 & 0 & 0 & 0 & 11 \\
\hline Morning Joe (Msnbc) & 2 & 1 & 0 & 0 & 0 & 0 & 0 & 0 & 3 \\
\hline The Washington Post & 3 & 2 & 0 & 0 & 1 & 0 & 0 & 0 & 6 \\
\hline CBS & 2 & 1 & 0 & 0 & 0 & 0 & 0 & 0 & 3 \\
\hline Otros & 0 & 0 & 0 & 0 & 0 & 0 & 0 & 0 & 0 \\
\hline Medios en general & 56 & 0 & 0 & 3 & 5 & 2 & 0 & 2 & 68 \\
\hline Total por palabra clave & 100 & 28 & 2 & 5 & 10 & 2 & 1 & 2 & 150 \\
\hline
\end{tabular}


media, o, menos a menudo, simplemente como media o the press- reciben un trato siempre negativo.

Pero cabe destacar que, más que elogiar a los supuestamente afines, Trump sobre todo critica a los que le son hostiles. Estas críticas se hacen en un estilo (tono y lenguaje) poco elaborado, llano, popular, lo que le configura el carácter auténtico y amateur, alejado de la profesionalización, observado ya en algún estudio (Enli, 2017).

Con su actitud respecto a los medios, Trump intensifica la polarización política del público, otra de las consecuencias de los cambios tecnológicos en el sector: la autoproducción y el autoconsumo (Castells, 2009) fomentan la polarización y el consumo de información autorreferencial; cuanto más sólida es la convicción de que la propia causa es la correcta, más probabilidad hay de que se busque sólo información que reafirme las propias preferencias. Por tanto, cuanto mayor es la polarización, mayor es la actitud selectiva de los seguidores, que a la vez acusan con mayor frecuencia y vehemencia a los medios convencionales (mainstream) de informar de manera sesgada cuando no avalan su posición apriorística, como denuncian Bennet e lyengar (2008).

\section{Trump reivindica el uso de sus redes so- ciales como canal de comunicación di- recto con el público}

Trump desmantela el orden institucional que prefigura unos medios independientes del poder político cuya función social es ejercer el control del Gobierno y los convierte en una herramienta a su servicio: los medios que les son afines son los suyos y los que no, de los otros. Los que le son críticos son mentirosos, deshonestos, falseadores, obedecen a intereses espurios y constituyen la "oposición" al poder. Lo decía explícitamente el 30 de enero:

"Where was all the outrage of the Democrats and the opposition party (the media) when our Jobs were fleeing our country?".

La actitud de Trump afecta, por tanto, el rol de los diversos actores que interactúan en el campo de la comunicación política, a saber: políticos, medios y ciudadanos. Así, el papel mediador de los medios se ve rechazado por Trump y, por el contrario, el de los ciudadanos y el suyo como político se ve reforzado. La actitud de Trump sería característica de un sistema mediático pluralista polarizado, siguiendo la taxonomía de Hallin y Mancini (2004), que es el propio de la Europa mediterránea; y se alejaría del modelo liberal, que los autores mencionados atribuyen a Estados Unidos y los países del Atlántico Norte. Un modelo de pluralismo polarizado que, como a su vez ya apuntaba López-García (2017, p. 576)

"quizá esté extendiéndose a otras latitudes; sin ir más lejos, en Estados Unidos".

Efectivamente, Trump degrada los medios plurales, independientes, centrados en la información y altamente profesionalizados, (The New York Times y CNN, entre otros) y exalta medios orientados a la opinión o el comentario, altamente politizados y con una clara toma de posición.
El tratamiento de Trump hacia la prensa contraviene los principios del periodismo como perro guardián o watchdog, por los cuales la prensa ejerce su papel fiscalizador ante el poder político. De esta manera, Trump se arroga la potestad de otorgar credibilidad a discreción: cuando los medios están a su favor, los elogia, les confiere credibilidad, y cuando están en contra los menosprecia, ataca o desconsidera, alegando que son fake news media.

\section{Más de un tercio de los tweets con refe- rencias a los medios incluyen la palabra fake}

Como señala Casero-Ripollés (2009), entre los mecanismos de control de la actividad periodística por parte de los actores políticos está la apelación directa al público, el denominado going public,

"donde los actores políticos se centran en evitar cualquier mediación periodística en la emisión del mensaje político" (Casero-Ripollés; Izquierdo-Castillo; Doménech-Fabregat, 2014, p. 28).

Con Twitter, Trump quiere evitar la mediación de los medios de comunicación. Reivindica la función y el poder de sus medios sociales como medio efectivo para llegar al pueblo norteamericano sin intervención de los medios convencionales. Para el presidente, por tanto, la real story es la que emana de sus redes sociales. El mejor y más concluyente ejemplo es este tweet del 29 de mayo:

"The Fake News Media works hard at disparaging \& demeaning my use of social media because they don't want America to hear the real story!".

Atribuirse la autoridad de ser el poseedor del relato verdadero refuerza la tendencia ya observada de que hemos dejado atrás la era de la sociedad de masas y estamos en la "sociedad personalmente mediatizada", en la que, frente a los antiguos contenidos elaborados por los mass media, tenemos a través de las redes

"la producción interactiva de información personalizada" (Bennet; lyengar, 2008, p. 723).

Ello hace presumir obsoleta no sólo la teoría de la aguja hipodérmica sino que cuestiona también la de la agenda-setting de McCombs y Shaw, como advirtieron ya en 2001 Chafee y Metzger, quienes predijeron que se pasaría de observar

"sobre qué temas quieren los medios que piense la gente" a observar "qué temas la gente dice a los medios sobre los que pensar" (Chafee; Metzger, 2001, p. 375).

Gracias a las redes sociales, pues, se reduce el protagonismo de los medios como mediadores entre los políticos y los ciudadanos, para dejar paso a un papel creciente del individuo, lo que tiene relación con el reciente auge del populismo: la actitud de Trump en Twitter contra los medios convencionales es un exponente más de la corriente populista que se ha manifestado en el ámbito de la comunicación política en los últimos años, que se caracteriza por una comunicación directa entre el líder y sus seguidores, cosa que sin duda Twitter facilita (Gainous; Wagner, 2014). 
Se observa también que en la comunicación de Trump vía Twitter y en relación con los medios, se cumplen dos de las características del estilo de comunicación populista (Jagers; Walgrave, 2007; Block; Negrine, 2017; Casero-Ripollés; Sintes-Olivella; Franch, 2017):

- la construcción de una identidad colectiva apelando al pueblo (Trump contrapone el pueblo a los mainstream media), y

- el antielitismo (Trump acusa a los medios convencionales de elitistas).

De esta manera, como observa Campos-Domínguez, lejos de ser un espacio en el que poder intercambiar puntos de vista y escuchar a los ciudadanos, los populistas utilizan Twitter

“para criticar a los críticos, llevar a cabo batallas personales y llamar la atención de los medios de comunicación" (Campos-Domínguez, 2017, p. 786).

\section{Agradecimientos}

Este artículo forma parte del proyecto financiado por el $\mathrm{Mi}$ nisterio de Economía, Industria y Competitividad español CSO2017-88620-P.

\section{Referencias}

Adams, Amelia; McCorkindale, Tina (2013). “Dialogue and transparency: A content analysis of how the 2012 presidential candidates use Twitter". Public relations review, v. 39, n. 4, pp. 357-359.

https://doi.org/10.1016/j.pubrev.2013.07.016

Aharony, Noa (2012). "Twitter use by three political leaders: an exploratory analysis". Online information review, v. 36, n. 4, pp. 587-603.

https://doi.org/10.1108/14684521211254086

Ammann, Sky L. (2010). "Why do they tweet? The use of Twitter by U.S. Senate candidates in 2010". Social science research network, December $15^{\text {th }}$.

http://dx.doi.org/10.2139/ssrn.1725477

Bennet, W. Lance; lyengar, Shanto (2008). "A new era of minimal effects? The changing foundations of political communication". Journal of communication, v. 58, pp. 707-731. https://doi.org/10.1111/j.1460-2466.2008.00410.x

Berger, Peter L.; Luckmann, Thomas (1967). The social construction of reality. A treatise in the sociology of knowledge. London: Penguin books. ISBN: 9780140135480 http://perflensburg.se/Berger\%20social-construction-ofreality.pdf

Block, Elena; Negrine, Ralph (2017). "The populist communication style: Toward a critical framework". International journal of communication, v. 11, pp. 178-197.

http://ijoc.org/index.php/ijoc/article/view/5820

Campos-Domínguez, Eva (2017). "Twitter y la comunicación política". El profesional de la información, v. 26, n. 5, pp. 785-793.

https://doi.org/10.3145/epi.2017.sep.01

Casero-Ripollés, Andreu (2009). "El control político de la información periodística". Revista latina de comunicación social, v. 64, pp. 354-366.
https://doi.org/10.4185/RLCS-64-2009-828-354-366

Casero-Ripollés, Andreu; Izquierdo-Castillo, Jéssica; Doménech-Fabregat, Hugo (2014). "From watchdog to watched dog: Oversight and pressures between journalists and politicians in the context of mediatization". Trípodos, v. 34, pp. 23-40.

http://www.tripodos.com/index.php/Facultat_Comunicacio_ Blanquerna/article/view/164

Casero-Ripollés, Andreu; Sintes-Olivella, Marçal; Franch, Pere (2017). "The populist political communication style in action: Podemos's issues and functions on Twitter during the 2016 Spanish general election". American behavioral scientist, v. 61, n. 9, pp. 986-1001.

https://doi.org/10.1177/0002764217707624

Castells, Manuel (2009). Communication power. Oxford, England: Oxford University Press. ISBN: 9780199567011

Corbin, Juliet; Strauss, Anselm (1990). "Grounded theory research: Procedures, canons, ans evaluative criteria". Qualitative sociology, v. 13, n. 1, pp. 3-21.

http://med-fom-familymed-research.sites.olt.ubc.ca/ files/2012/03/W10-Corbin-and-Strauss-grounded-theory.pdf

Coromina, Òscar (2017). "The struggle for the story in political disputes. The case of the $9 \mathrm{~N}$ participation process". EI profesional de la información, v. 26, n. 5, pp. 884-893.

https://doi.org/10.3145/epi.2017.sep.10

Chaffee, Steven H.; Metzger, Miriam J. (2001). "The end of mass communication?". Mass communication and society, v. 4, n. 4, pp. 365-379.

https://goo.gl/wu5ArJ

https://doi.org/10.1207/S15327825MCS0404_3

Enli, Gunn-Sara (2017). "Twitter as arena for the authentic outsider: Exploring the social media campaigns of Trump and Clinton in the 2016 US presidential election". European journal of communication, v. 32, n. 1, pp. 50-61. https://doi.org/10.1177/0267323116682802

Enli, Gunn-Sara; Skogerbø, Eli (2013). “Personalized campaigns in party-centred politics: Twitter and Facebook as arenas for political communication". Information, communication \& society, v. 16, n. 5, pp. 757-774.

https://doi.org/10.1080/1369118X.2013.782330

Gainous, Jason; Wagner, Kevin M. (2014). Tweeting to power: The social media revolution in American politics. Oxford, England: Oxford University Press. ISBN: 978019 9965076

Golbeck, Jennifer; Grimes, Justin M.; Rogers, Anthony (2010). "Twitter use by the U.S. Congress". Journal of the American Society for Information Science and Technology, v. 61, n. 8, pp. 1612-1621.

https://bit.ly/2PlqjzA

https://doi.org/10.1002/asi.21344

Graham, Todd; Jackson, Daniel; Broersma, Marcel (2016). “New platform, old habits? Candidates' use of Twitter during the 2010 British and Dutch general election campaigns". New media \& society, v. 18, n. 5, pp. 765-783.

https://doi.org/10.1177/1461444814546728 
Hallin, Daniel C.; Mancini, Paolo (2004). Comparing media systems: Three models of media and politics. Cambridge, England: Cambridge University Press, 358 pp. ISBN: 9780 521543088

Hernández-Santaolalla, Víctor; Rubio-Hernández, Maríadel-Mar (2017). “Fandom político en Twitter: La Cueva y los partidarios de Alberto Garzón en las elecciones generals españolas de 2015 y 2016". El profesional de la información, v. 26, n. 5, pp. 838-849.

https://doi.org/10.3145/epi.2017.sep.06

Jackson, Nigel; Lilleker, Darren (2011). “Microblogging, constituency service and impression management: UK MPs and the use of Twitter". Journal of legislative studies, v. 17, n. 1, pp. 86-105.

https://doi.org/10.1080/13572334.2011.545181

Jagers, Jan; Walgrave, Stefaan (2007). “Populism as political communication style: An empirical study of political parties' discourse in Belgium". European journal of political research, v. 46, n. 3, pp. 319-345.

https://doi.org/10.1111/j.1475-6765.2006.00690.x

Jungherr, Andreas (2016). "Twitter use in election campaigns: A systematic literature review". Journal of information technology \& politics, v. 13, n. 1, pp. 72-91.

https://doi.org/10.1080/19331681.2015.1132401

Kahn, Kim-Fridkin; Kenney, Patrick J. (2002). "The slant of the news. How editorial endorsements influence campaign coverage and citizens' views of candidates". American political science review, v. 96, n. 2, pp. 381-394.

https://seminariodeinvestigadoresuptns. files.wordpress. com/2014/11/kahn-kenney-2002.pdf

https://doi.org/10.1017/S0003055402000230
López-García, Guillermo (2017). “Comunicación política y discursos sobre el poder". El profesional de la información, v. 26, n. 4 , pp. 573-578.

https://doi.org/10.3145/epi.2017.jul.01

Miles, Matthew B.; Huberman, A. Michael (1994). Quality data analysis. An expanded sourcebook. Thousand Oaks (CA): Sage Publications. ISBN: 9780803955400

Newman, Nic; Fletcher, Richard; Kalogeropoulos, Antonis; Levy, David A. L.; Nielsen, Rasmus-Kleis (2018). Reuters Institute. Digital news report 2017. Reuters Institute for the Study of Journalism. Reuters Institute \& University of Oxford.

https://reutersinstitute.politics.ox.ac.uk/sites/default/files/ Digital\%20News\%20Report\%202017\%20web_0.pdf

Ott, Brian L. (2017). "The age of Twitter: Donald J. Trump and the politics of debasement". Critical studies in media communication, v. 34, n. 1, pp. 59-68.

https://doi.org/10.1080/15295036.2016.1266686

Small, Tamara A. (2011). "What the hashtag? A content analysis of Canadian politics on Twitter". Information communication \& society, v. 14, n. 6, pp. 872-895.

https://doi.org/10.1080/1369118X.2011.554572

Stolee, Galen; Caton, Steve (2018). "Twitter, Trump and the base: a shift to a new form of presidential talk?". Signs and society, v. 6, n. 1, pp. 147-165.

https://doi.org/10.1086/694755

Sulzberger Jr., Arthur; Baquet, Dean (2016). "To our readers, from the publisher and the executive editor". The New York Times, November $13^{\text {th }}$.

https://www.nytimes.com/2016/11/13/us/elections/toour-readers-from-the-publisher-and-executive-editor.html

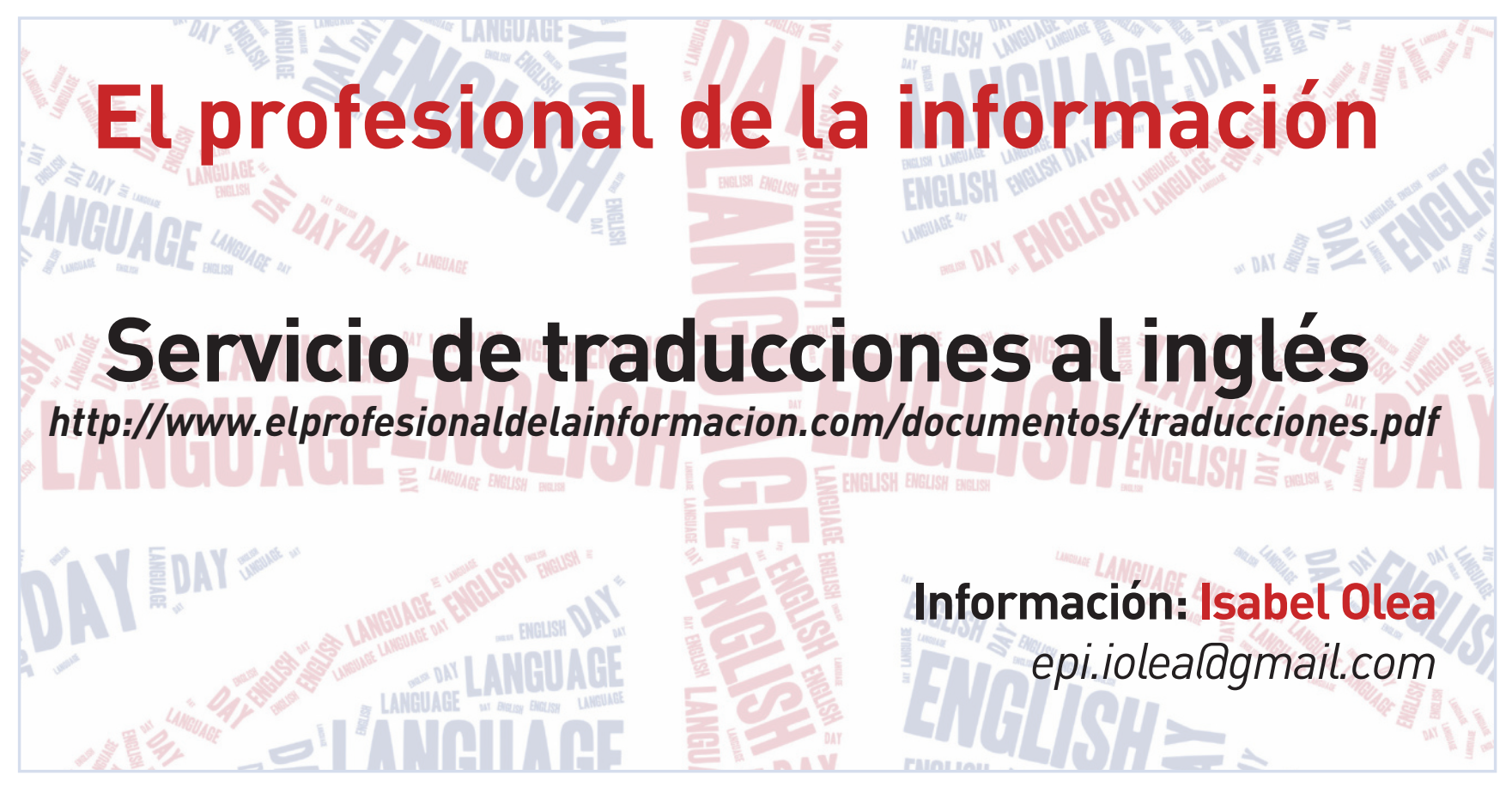

